\title{
Uncovering the link between malfunctions in Drosophila neuroblast asymmetric cell division and tumorigenesis
}

\author{
Corey Kelsom and Wange Lu*
}

\begin{abstract}
Asymmetric cell division is a developmental process utilized by several organisms. On the most basic level, an asymmetric division produces two daughter cells, each possessing a different identity or fate. Drosophila melanogaster progenitor cells, referred to as neuroblasts, undergo asymmetric division to produce a daughter neuroblast and another cell known as a ganglion mother cell (GMC). There are several features of asymmetric division in Drosophila that make it a very complex process, and these aspects will be discussed at length. The cell fate determinants that play a role in specifying daughter cell fate, as well as the mechanisms behind setting up cortical polarity within neuroblasts, have proved to be essential to ensuring that neurogenesis occurs properly. The role that mitotic spindle orientation plays in coordinating asymmetric division, as well as how cell cycle regulators influence asymmetric division machinery, will also be addressed. Most significantly, malfunctions during asymmetric cell division have shown to be causally linked with neoplastic growth and tumor formation. Therefore, it is imperative that the developmental repercussions as a result of asymmetric cell division gone awry be understood.
\end{abstract}

Keywords: Asymmetric cell division, Neuroblasts, Polarity, Determinants, Spindle orientation, Tumorigenesis

\section{Introduction}

Asymmetric cell division is a phenomenon that has long been studied, especially in the developing nervous system of invertebrates and vertebrates. Asymmetric cell division is a mechanism whereby any given cell divides to give rise to two daughter cells, each of which possesses a different fate than the other [1]. Such "fates" can be manifested as differences in size, morphology, gene expression pattern or the number of subsequent cell divisions undergone by the two newly born daughter cells [1].

To date there are two established modes of asymmetric cell division. One type of division, commonly referred to as a niche-controlled, or extrinsic, mechanism of cell division, emphasizes the importance of the stem cell niche (Figure 1A) [2]. Environmental factors influence

\footnotetext{
* Correspondence: wange.lu@med.usc.edu

Department of Biochemistry and Molecular Biology, Eli and Edythe Broad Center for Regenerative Medicine and Stem Cell Research, University of Southern California, 1425 San Pablo Street, Los Angeles, CA 90033, USA
}

the ability to maintain the progenitor population, and a cell relies on contact with its stem cell niche to be able to self-renew. A second, intrinsic mechanism of asymmetric cell division serves as the dominant mode of division during development and will be the focus of this discussion rather than the niche-controlled mechanism (Figure 1B). With regard to the intrinsic mechanism, regulators of self-renewal are asymmetrically localized during mitosis, so that when cells divide only one daughter cell inherits these regulators and thus takes on a different fate than its sister cell [3,4]. Actively dividing Drosophila neuroblasts, which serve as precursor and progenitor cells of the nervous system, take the intrinsic route of asymmetric cell division. A brief background of Drosophila neural progenitor cells will be given in this review. Notch signaling, which is a very important component that ties into the developmental process of neurogenesis, will also be discussed.

The major aspects of asymmetric cell division in Drosophila will be discussed at length. An apical-basal axis of polarity is set up within cells, which is used to both 


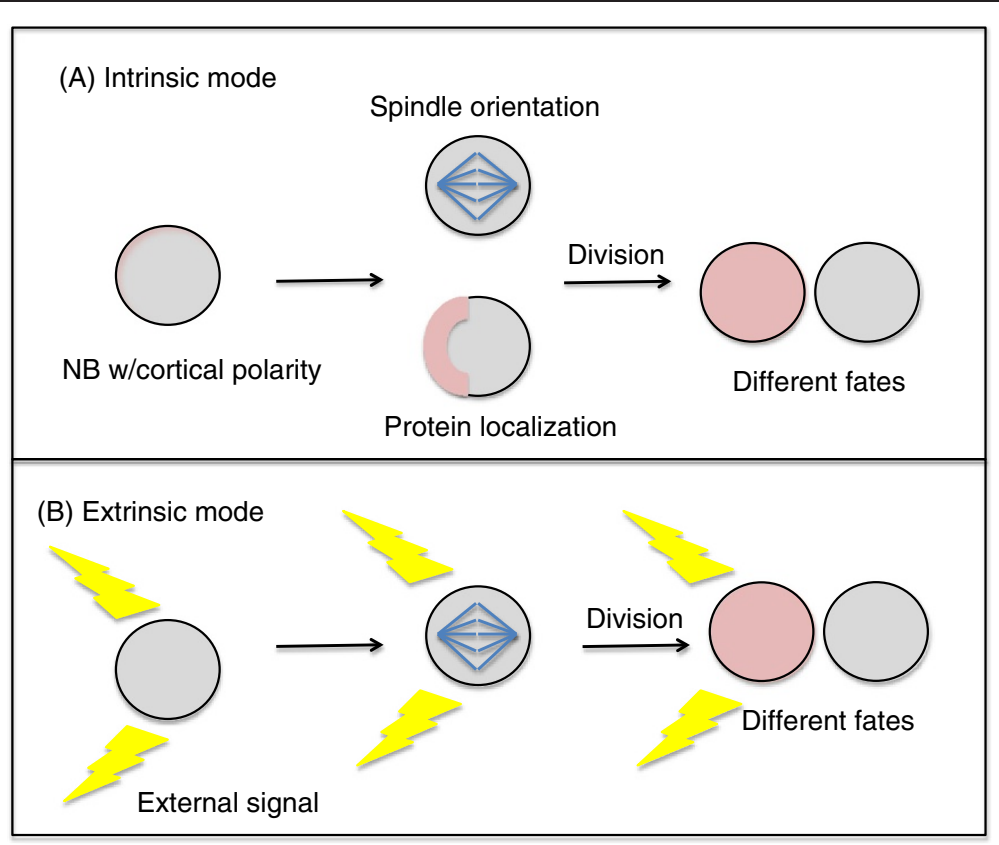

Figure 1 Intrinsic vs. extrinsic modes of asymmetric cell division. (A). During the intrinsic mode of asymmetric division, cells such as Drosophila neuroblasts possess an inherent axis of polarity. This polarity allows certain proteins such as cell fate determinants to localize asymmetrically within the cells. The mitotic spindle orients itself to be along the same axis of polarity, so when cellular division occurs, only one daughter cell receives the aforementioned determinants. Each daughter cell thus has a different fate. (B) During the extrinsic mode of asymmetric division, cellular precursors receive external, or extracellular, signals to self-renew (yellow). The mitotic spindle is oriented perpendicular to these external signals. When cellular division occurs, only one of the daughter cells continues to receive these signals and the two cells therefore have different fates.

asymmetrically distribute self-renewal determinants and orient the mitotic spindle to polarize the determinants, is a very important feature of asymmetric cell division. The cell fate determinants of neural stem cell self-renewal and their asymmetric localization are also essential in ensuring that the divisional machinery operates correctly. Additionally, the role that mitotic spindle orientation plays in asymmetric division is tantamount to this developmental process and will also be discussed. The coordination of asymmetric protein localization with cell cycle progression is another aspect of asymmetric cell division that will be covered as well. Moreover, of great importance to this field of research is the concept that failure of asymmetric cell division to occur properly has widespread consequences, mainly that of neoplastic cellular growth and tumorigenesis. This review will discuss the developmental outcomes faced by dividing neuroblasts in Drosophila when asymmetric cell division machinery is altered or lost. In particular, the repercussions of disruption of cortical polarity due to missegregated cell fate determinants and proteins, as well as misalignment of the mitotic spindle, will be discussed. Existence of this undeniable link between asymmetric cell division gone awry and tumorigenesis shows that understanding the mechanisms behind asymmetric cell division hold great value not only on a developmental basis, but on the clinical level as well.

\section{Neural progenitor cells}

Neuroblasts serve as the progenitor cell population in the developing Drosophila nervous system, and demonstrate the importance of asymmetric cell division in generating terminally differentiated neurons and glia. There are two types of neuroblasts in the developing nervous system - embryonic neuroblasts, which give rise to the simple nervous system present in larva, and larval neuroblasts, which generate the neurons in the fly's adult nervous system $[5,6]$.

Neuroblasts initially divide symmetrically - one neural progenitor cell divides to produce two identical, daughter neuroblasts, thereby maintaining and expanding the population of neural stem cells (Figure 2A) [5,6]. As neural development progresses, neuroblasts then undergo asymmetric cell divisions. During division, one daughter cell is produced that is identical to its parent, and therefore maintains a neuroblast identity. The second daughter that is generated is smaller in size and is referred to as a ganglion mother cell (GMC). Ganglion mother cells proceed to undergo one last division to ultimately generate two differentiating neurons. As will be discussed 


\section{(A) Wild type dividing NB}
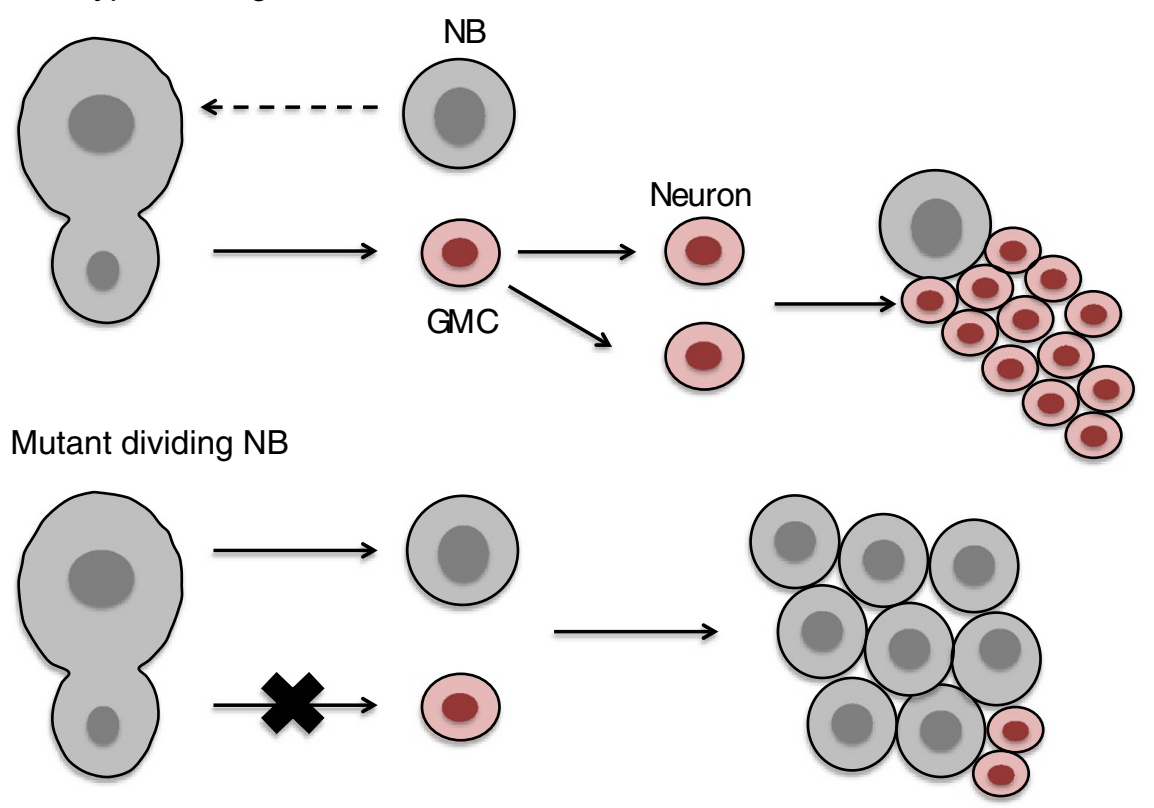

Figure 2 Neuroblast self-renewal vs. differentiation and tumorigenesis. (A) A wild type neuroblast divides to form two daughter cells, one of which becomes a self-renewing neuroblast (gray) and one of which becomes a ganglion mother cell (GMC) (red). The GMC divides terminally to become differentiated neurons. There is a balance between self-renewing neuroblasts and neurons. (B) A mutant neuroblast (such as Miranda knockouts or Pins, Lgl double knockouts) fail to divide asymmetrically and create only self-renewing neuroblasts. This results in an unrestricted growth of neural precursors at the expense of differentiated neurons, thus leading to neoplastic growth and tumor formation.

below, actively dividing mutant neuroblasts fail to generate GMCs, resulting in the inappropriate accumulation of daughter neuroblasts at the expense of neurons (Figure 2B). In many instances this has dire effects on development.

\section{The role of notch signaling during neurogenesis}

The Notch signaling pathway has been shown to function as a key regulator in the developing nervous system. Findings from numerous studies contribute to the fact that Notch signaling controls the balance between self-renewal and differentiation of neural progenitor cells [7-9], and elegantly coordinates neuroblastic asymmetric cell division. Neuronal differentiation is triggered by transcriptional activators such as Mash1 and Neurogenin2 (Ngn2) [10,11]. Mash1 and Ngn2 simultaneously activate the expression of the Notch receptor ligands Delta1, which activates Notch in neighboring cells. Neuralized (Neur) is an E3 ubiquitin ligase protein that facilitates the endocytosis of Delta and the extracellular domain of Notch [12-16]. Notch activation is then followed by nuclear transport of the Notch intracellular domain (NICD) and subsequent formation of a transcription activator complex [17]. This complex triggers the activation of Hes1 and Hes5, which act to repress the expression of proneural genes in neighboring progenitor cells [18]. This achieves the purpose of asymmetric cell division: it ensures the formation of differentiating neurons while simultaneously allowing neighboring cells to remain as neural progenitors.

Updates to the study of Notch signaling and its role in asymmetric cell division of neuroblasts have recently been made. Monastirioti and colleagues have identified Drosophila Hey as a target of Notch during neurogenesis [19]. Hey is a basic-helix-loop-helix-Orange (bHLH-O) transcription factor that is expressed primarily in the population of neuroblasts possessing activated Notch signaling, and therefore is thought to contribute to maintaining/expanding the neural progenitor cell population during development [19]. A recent study has also investigated other candidate genes that Notch signaling regulates in the context of neurogenesis. A gene referred to as Deadpan (Dpn) that encodes a bHLH transcription factor is another direct target of the Notch signaling pathway; so far this has only been demonstrated in intermediate progenitor cells [20]. Overexpression of Dpn in INPs gives rise to a cancer-like phenotype in which neuroblasts overproliferate inappropriately [20].

The first study to show the link between both asymmetric division machinery and Notch signaling and specification of a neurotransmitter neuronal phenotype was performed in Drosophila by Tio et al [21]. Findings from this study showed that loss of function of the proteins Inscuteable and Bazooka resulted in an 
excess number of dopaminergic neurons. On the other hand, loss of function of basally distributed proteins such as Numb resulted in a reduction of dopaminergic neurons [21]. Additionally, loss of Notch signaling results in an excess amount of this neuronal phenotype, and vice versa. Given the high levels of conservation between Drosophila and higher-level organisms, this study may hold great promise in understanding the link between asymmetric division and neuronal specification.

Notch signaling regulates a vast amount of developmental processes in Drosophila, including that of optic lobe development [22]. Briefly, loss of function analyses of both Notch and Delta demonstrate that Notch signaling might play a dual role here: maintenance of neuroepithelial stem cell population and inhibition of these stem cells toward differentiation into medulla neuroblasts [22].

\section{Cell-fate / segregating determinants}

Segregating determinants, also referred to as cell-fate determinants, are proteins that play a crucial role in specifying daughter cell fate (Table 1, Figure 3 ). It is the asymmetric localization of these particular determinants (in addition to other factors) to the basal side of the dividing neural progenitor cell that is largely thought to produce two daughter cells, each with a different fate.

\section{Numb}

Numb is a transcription factor that was originally identified in Drosophila sensory organ precursor (SOP) cells and has been demonstrated to segregate asymmetrically in neuroblasts [23]. Numb has been shown to serve as a tissue-specific repressor of the Notch signaling pathway $[24,65]$; it binds alpha-adaptin and potentially plays a role in directing intracellular transport of Notch intermediates [66]. Loss or disruption of Numb function in the larval brain manifests in the overproliferation of mutant neuroblasts, which therefore gives rise to a tumor-like phenotype $[25,26]$.

More recently, the role of additional proteins in regulating the asymmetric localization of Numb has been investigated. Wang and colleagues demonstrated that protein phosphatase $2 \mathrm{~A}$ (PP2A) is a brain tumor suppressor protein that forms a heterotrimeric complex that functions to inhibit the self-renewal of neuroblasts [27]. The PP2A complex regulates, among other things, the asymmetric localization and phosphorylation of Numb [27]. Additionally, the Hem/Kette/Nap1 protein has been shown to play a very important role in the asymmetric division of Drosophila neuroblasts; it does so by regulating the localization of Numb and another adaptor protein known as Inscuteable [38]. Hence, Hem/Kette/ Nap1 mutant GMCs display symmetric, rather than asymmetric division. Neur, a protein previously established to play a role in Notch signaling, has recently

Table 1 Key players in asymmetric cell division

\begin{tabular}{|c|c|c|c|}
\hline $\begin{array}{l}\text { Asymmetric division } \\
\text { protein }\end{array}$ & Protein function & Phenotype associated with mutation & References \\
\hline Numb & Neuronal differentiation & NB overproliferation & {$[23-27]$} \\
\hline Pon & Neuronal differentiation & Delocalization of Numb & [28] \\
\hline Brat & $\begin{array}{l}\text { Regulates Prospero localization } \\
\text { Inhibits translation }\end{array}$ & Delocalization of Prospero; NB overproliferation & {$[29-32]$} \\
\hline Miranda & Localization of basal proteins & Delocalization of basal proteins; NB overproliferation & {$[3,33]$} \\
\hline Prospero & Neuronal differentiation & NB overproliferation & {$[29-31,34-37]$} \\
\hline Staufen & Localization of Prospero mRNA & Delocalization of Prospero mRNA & {$[3]$} \\
\hline \multirow[t]{2}{*}{ Pins, Gai, Loco } & Spindle orientation & \multirow{3}{*}{$\begin{array}{l}\text { Delocalization of basal proteins; } \\
\text { NB overproliferation }\end{array}$} & \multirow[t]{3}{*}[38-46]{} \\
\hline & Localization of basal proteins & & \\
\hline Inscuteable & $\begin{array}{l}\text { Links the heterotrimeric } \mathrm{G} \text { protein complex } \\
\text { with the Par complex }\end{array}$ & & \\
\hline aPKC, Bazooka/Par3, Par6 & Maintaining apical and basal polarity & Apical and basal polarity defects & {$[28,32,33,47-57]$} \\
\hline Mud & Spindle orientation & Spindle misorientation; NB overproliferation & {$[39-46,56,58,59]$} \\
\hline Lgl (cortical localization) & Localization of basal proteins & Delocalization of basal proteins; NB overproliferation & {$[32,49-54]$} \\
\hline Aurora A (centrosome) & Maintaining apical/basal polarity & \multirow{3}{*}{$\begin{array}{l}\text { Apical and basal polarity defects; spindle } \\
\text { misorientation, NB overproliferation }\end{array}$} & \multirow[t]{3}{*}[25,26,60-64]{} \\
\hline Polo (centrosome) & Spindle orientation & & \\
\hline PP2A (cytoplasmic) & & & \\
\hline Dpn & NB specification & Loss of NB & {$[20]$} \\
\hline Zif & Apical/basal polarity & Apical and basal polarity defects; NB overproliferation & {$[55]$} \\
\hline
\end{tabular}

Cells are colored based on localization. Green denotes basal localization, blue denotes apical localization, and purple indicates nuclear localization. 


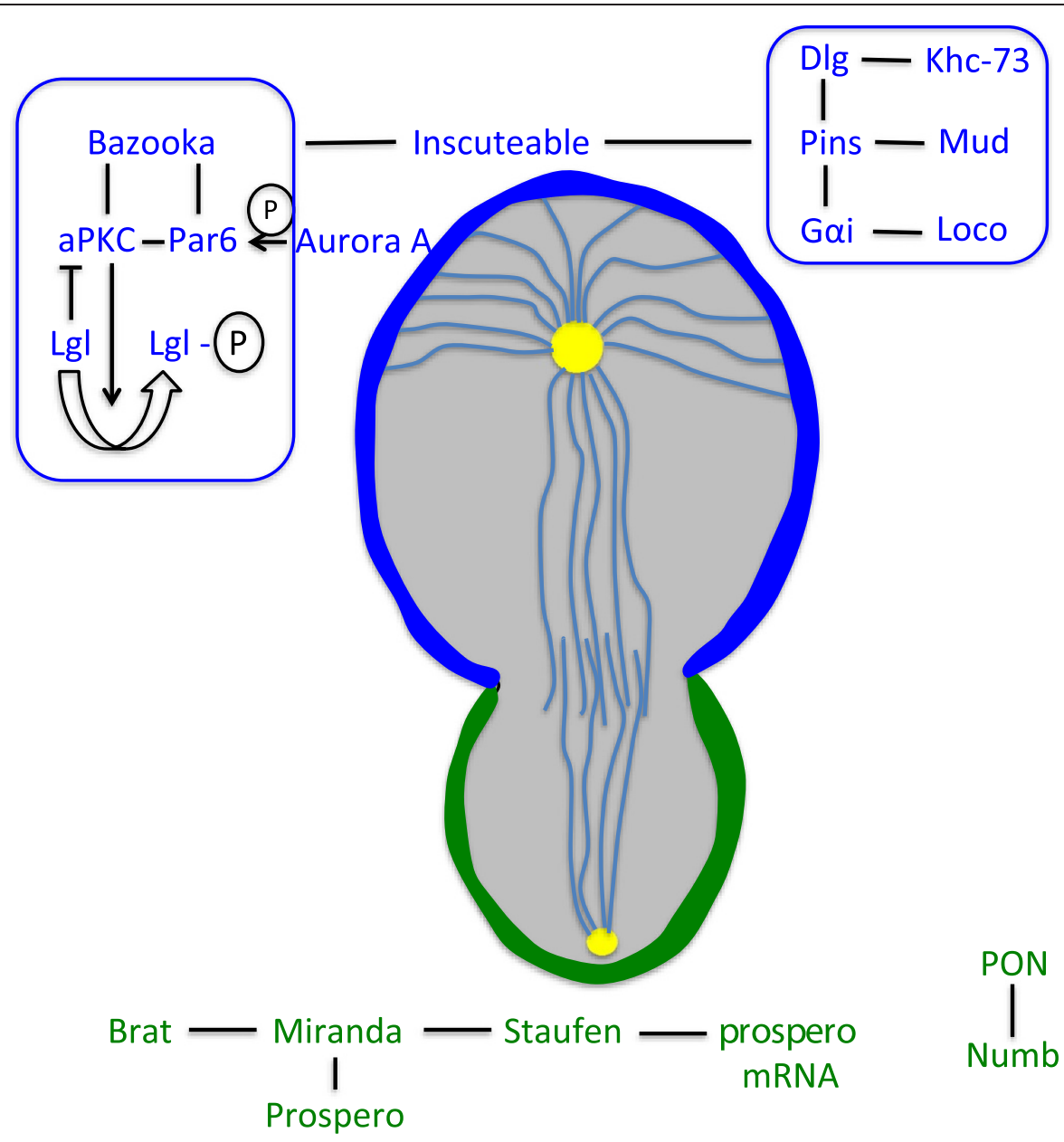

Figure 3 Some of the key players in asymmetric cell division of Drosophila neuroblasts. Asymmetric localization of proteins in dividing neuroblasts. Basal proteins are visualized in green, while apically localized proteins are visualized in blue. Apical proteins Bazooka, aPKC, and Par-6 form a complex whose responsibility is to establish cell polarity. There is a second complex of apical proteins, which consists of Gai, Pins, and Loco. This complex is linked to Baz-aPKC-Par-6 by another protein known as Inscuteable. Aurora-A phosphorylates Par-6 to control aPKC's substrate specificity. The proteins Miranda, Brat and Prospero form a complex that is basally located. PON and Numb are two other basal proteins that form a separate complex; both complexes work to regulate differentiation of the GMC.

been shown to promote the asymmetric localization of Numb by downregulating expression of the transcription factor Pdm1 [67]. This function is evidenced by mutational analaysis, which shows that Numb is symmetrically (rather than asymmetrically) localized in Neur mutants. Moreover, Neur overexpression results in expansion of the neuroblast population at the expense of differentiating neurons [67].

\section{Prospero}

A second cell-fate determinant that has also been shown to segregate asymmetrically in neuroblasts is the transcription factor Prospero. Choksi and colleagues have demonstrated on the genome-wide level that Prospero (Pros) has several hundred binding sites in the Drosophila genome [34]. Importantly, this study showed that Pros acts as a "switch" between neuroblast self-renewal and differentiation: it has the ability to repress neuroblast and cell-cycle genes, as well as regulate neural differentiation genes [34].

Pros mutant GMCs fail to commit to a differentiated, neuronal fate: these mutant cells have prolonged expression of neuroblast markers and inappropriately continue to divide [34]. It had previously been postulated that the upregulation of cell cycle regulators, mainly Cyclin A, Cyclin E, and Cdc25, may be the reason for this occurrence in Pros mutant neuroblasts [35]. More recently, Berger et al have demonstrated that Cyclin E possesses cell-cycle independent roles in asymmetric division: it inhibits Pros function, and may also serve to regulate the cortical localization of Pros, hence allowing neuroblasts to maintain their identity rather than committing to the neuronal lineage [36]. By regulating Pros localization, CycE therefore plays a crucial role in 
maintaining the neural progenitor population. Similar to Numb, mutational analysis shows that Pros mutations give rise to stem cell-derived tumors in larval neuroblasts [29-31].

In addition to its role as a cell fate determinant in GMCs, Pros also possesses a role in coupling cell cycle progression to neurogenesis during development: its transient expression ensures that neuronally-committed cells exit the cell cycle at the appropriate time [37].

\section{Brat}

Brat is the third cell-fate determinant and growth inhibitor that was discovered to play a role in regulating the balance between neuroblast self-renewal and differentiation [29-31]. During neural development, Brat (in combination with Pros) segregates asymmetrically into only one of two daughter neuroblasts to specify GMC fate [30]. Similar to Numb, loss of Brat results in both daughter cells taking on a neuroblast identity, which ultimately gives rise to a tumor phenotype [30]. Further mutational analyses have demonstrated that the phenotype of Pros/Brat double mutants is one that lacks most, if not all, GMCs due to an overexpansion of neuroblasts [30]. Observations of these mutant phenotypes have led to the speculation that Brat may function to inhibit cell growth in one of two newly born neuroblast daughters so as to generate one neuron and one neuroblast, rather than two neuroblasts [32]. The true molecular mechanism by which Brat operates, however, remains to be clarified.

\section{Adaptor proteins}

While proper function of the segregating determinants is crucial for asymmetric cell division, adaptor proteins are just as important in ensuring that division is properly executed. Adaptor proteins facilitate the asymmetric localization of Numb, Pros and Brat.

\section{Miranda}

Miranda serves as the adaptor protein for segregating determinants Pros, Brat, and Staufen, although Staufen's functions will not be covered in this review. For more details regarding Staufen, refer to Betschinger and Knoblich [3]. Miranda behaves similarly to Pros and Brat; in dividing neuroblasts it too localizes asymmetrically and segregates into one of the two daughter cells [3]. Importantly, when Miranda is mutated, both Pros and Brat segregate symmetrically rather than asymmetrically in dividing neuroblasts, and the cell-fate determinants are therefore uniformly cytoplasmic.

Atwood and colleagues have more recently shown that atypical protein kinase $(\mathrm{aPKC})$, which is a regulator of cell polarity, directly phosphorylates Miranda [33]. Phosphorylation displaces Miranda from the apical cortex, where it can then work to polarize the cell-date determinants. These findings counter the theory that Miranda is regulated by a more complicated cascade involving aPKC, Lgl and myosin II [33]. Regardless of the mechanism by which it works, Miranda is a crucial adaptor protein that connects Pros and Brat to the machinery for asymmetric protein localization.

\section{Pon}

Pon, which stands for Partner of Numb, is so-named because of its function: it is the adaptor protein for Numb and therefore binds to Numb. Unlike Miranda, however, Pon is not required for the asymmetric localization of Numb [28]. In the absence of Pon, localization of Numb is delayed in metaphase, which therefore results in a defect in the neuroblast self-renewal [28].

\section{Setting up polarity}

While the asymmetric localization of cell-fate determinants and the functions they play in neuroblast selfrenewal or neuronal commitment has been established, the question of how they are directed to the basal cortex remains to be answered. The answer lies within an axis of polarity that is set up during interphase. Cell-fate determinants and the orientation of stem cell division both take instruction from this axis of polarity, which consists of aPKC and the Par proteins Par-3 (Bazooka in Drosophila) and Par-6 (Figure 3) [47,48].

Par-3, Par- 6 and aPKC are required for establishing apical-basal polarity in developing neuroblasts: they concentrate to the apical cell cortex of the neuroblast [32]. The localization of these proteins is opposite of the location that the cell-fate determinants concentrate in mitosis, and their presence ensures that the determinants are segregated into the basal cell cortex. When any one of the three polarity proteins is mutated, the cell-fate determinants are distributed uniformly in the cell cortex, and mitotic spindles orient randomly $[47,48]$. Hence, aPKC and the Par proteins are critical for constructing a "blue-print", in which the cell-fate determinants are properly distributed and the mitotic spindle is properly oriented.

Due to the extensive amount of literature in existence concerning the role of the Par proteins, this discussion will mainly emphasize the role that Lgl, the substrate of aPKC, plays in the asymmetric localization of segregating determinants.

Lethal (2) giant larvae (abbreviated Lgl) was identified by several groups as the key substrate for aPKC [49-51]. Unlike the other polarity proteins that are apically localized, Lgl is uniformly distributed throughout the cortex. Lgl is necessary for ensuring that the cell-fate determinants are brought to the cell cortex and localized asymmetrically during mitosis [52,53]. The mechanism 
by which $\mathrm{Lgl}$ is able to achieve its purpose was elucidated soon after, and concerns the Par proteins, mainly aPKC. aPKC is responsible for phosphorylating $\mathrm{Lgl}$ on three conserved serines at the cell cortex [49]. This phosphorylation event is proposed to prevent Lgl from associating with the actin skeleton, and most significantly, prevents the cell fate determinants from localizing apically [49]. aPKC-mediated phosphorylation of Lgl seems to somehow inactivate $\mathrm{Lgl}$, and is evidenced by the phenotype of aPKC overexpression, which resembles that of Lgl loss of function.

Adding more complexity to the puzzle is the fact that aPKC also possesses the ability to phosphorylate segregating determinants directly, rather than acting on $\mathrm{Lgl}$ first. Smith and colleagues have demonstrated that aPKC can directly phosphorylate Numb, whereby Numb is transported from the cell cortex into the cytoplasm [54]. This study demonstrated, utilizing a form of Numb lacking two protein kinase $\mathrm{C}$ (PKC) phosphorylation sites, that inability to phosphorylate Numb results in the inappropriate accumulation of Numb at the cell membrane and unresponsiveness to PKC activation [54].

In a 2008 review, Knoblich has proposed a model to account for aPKC and Lgl activity in neuroblasts, whereby aPKC, which is localized at the apical cortex, functions to restrict Lgl to the basal side of the neuroblast [32]. Given Lgl's responsibility of recruiting the cell-fate determinants to the cortex, this makes sense, since the determinants only localize basally. While this model is certainly logical, the molecular mechanism by which Lgl operates has not yet been elucidated.

Recent studies have delved into mechanisms underlying aPKC function. Chang et al, for instance, have shown that Zif is a transcription factor and that Zif is required for aPKC to both be expressed and asymmetrically localized [55]. Zif acts to directly repress transcription of aPKC, and in turn, aPKC phosphorylates Zif, which ultimately leads to Zif inactivation in neuroblasts. The combined actions of these two proteins thus play an indispensable role in setting up cortical polarity and also controlling progenitor self-renewal [55].

\section{Importance of proper mitotic spindle orientation}

The significance of mitotic spindle orientation in regulating neuroblast division has also been extensively studied and renewed $[68,69]$. Here, the coordination between mitotic spindle orientation and asymmetric localization of the segregating determinants will be discussed.

Kraut and colleagues first determined that a protein known as Inscuteable (Insc) plays an immensely important role in coordinating mitotic spindle alignment and localization of the cell-fate determinants to the basal cortex in dividing neuroblasts [70]. Inscuteable operates by binding the polarity protein Bazooka (Par-3) in the apical region of neuroblasts, and recruits another protein called Pins (which will be discussed below) [39]. Zhu and Bhat have recently shown that the Drosophila protein Hem/Kette/Nap1 also regulates localization of Inscuteable. As was previously discussed, this protein regulates the asymmetric division of neural progenitors by controlling Numb localization [38].

It is important to note that the binding of Insc to Pins triggers the activation of two downstream pathways that participate in mitotic spindle positioning, both of which are mediated by Pins. The first pathway is often referred to as the Pins-Mud pathway. Pins is a protein whose structural features are functionally purposeful: it contains three domains called GoLoco domains in its Cterminal region; these domains bind Goi, which is a heterotrimeric G protein subunit [39]. Upon binding, Pins is recruited to the plasma membrane and switches from and switches from an inactive to active state. It is in this active state that the $\mathrm{N}$-terminal region of Pins binds to another protein called Mud (which stands for Mushroom body defect) [40-42]. Mud is the Drosophila homolog of NuMA, and is thought to play a role in recruiting the Dynein/Dynactin complex [43]. This complex functions to generate pulling forces on astral microtubules so as to further advance mitotic spindle positioning [43].

The second pathway that is activated upon Insc binding Pins is the Dlg pathway [44,45]. In a 2005 study, Siegrist and Doe elegantly showed that astral microtubules bind to a kinesin referred to as Khc-73 as well as the protein Discs large (Dlg). Of note is that the Dlg pathway functions during metaphase to coordinate neuroblast polarity with the mitotic spindle, independent from the Pins-Mud pathway [45].

Recently, others have further investigated the mechanisms by which Inscuteable exerts its effects on mitotic spindle positioning through Pins. Results from a study by Mauser and Prehoda have suggested that Insc preferentially inhibits the Mud pathway, while enabling continued activation of the Dlg pathway [46]. A variety of rationales may explain these findings, one of them being assurance that the spindle is attached to the cortex via Dlg before spindle pulling forces are activated via the Mud pathway [46].

A 2011 study has characterized the Drosophila cytoplasmic polyadenylation element binding (CPEB) protein Orb2. CPEB proteins function to bind mRNAs in order to control their localization and subsequent translation. Hafer and colleagues report that Orb2 functions in the asymmetric division of both stem and precursor cells in the context of the developing Drosophila nervous system [56]. Additionally, Orb2 mutants present with disrupted mitotic spindle alignment; results from this study suggest that it may serve to promote the localization of aPKC [56]. 
Speicher et al were first to demonstrate that the PDZ protein Canoe (Cno) plays a role in both the localization of cell-fate determinants and orientation of the mitotic spindle in asymmetrically dividing neuroblasts [58]. Cno apically localizes with the Bazooka/Par-3 in neuroblasts, and was also found to be essential for proper distribution of cell-fate determinants on the basal side of the cell; importantly, failure of the determinants to basally distribute resulted in misorientation of the mitotic spindle. This study further demonstrated that Cno interacts with the proteins Inscuteable, Goi, and Mud, and acts downstream of apical proteins Insc-Pins-Goi, but upstream of Mud [58]. More recently confirming Cno's involvement in regulating mitotic spindle orientation and neuroblast cortical polarity is a study demonstrating that Rap1, a Ras-like small guanosine triphosphatase, signals through $\mathrm{Cno}$ and another guanine nucleotide exchange factor known as Rgl in order to regulate neuroblast polarity [59]. Carmena and colleagues postulate that Rap1 forms a novel Rap1-Rgl-Ral signaling network that interacts with other apical proteins to influence neuroblast cortical polarity and spindle orientation: loss of function of Rap1, Rgl and Ral proteins affect both spindle orientation and the localization of proteins Mud and Cno [59].

\section{Consequences of disrupting asymmetric cell division - neoplastic growth and tumorigenesis}

It has long been postulated that tumorigenesis and uncontrolled cellular replication may be causally linked to asymmetric cell division gone awry. To date, transplantation studies in Drosophila (as well as in mammals) remain the most reliable method for assessing the neoplastic cellular growth of cells in the context of experimentally induced tumors [71]. When tissue possessing mutant forms of the proteins central to asymmetric neuroblast division, such as Miranda, Prospero, Numb, Lgl, Brat and Pins, is transplanted into the wild-type Drosophila hosts, excessive overgrowth is observed; this massive cellular overgrowth is lethal to the organism $[72,73]$. Further analysis shows that the phenotypes observed from these transplantation experiments resemble malignant, neoplastic growth. The tumors that grow in these organisms have the capacity to be reimplanted into successive healthy host organisms for a number of years, demonstrating that these cells are immortalized [73]. Importantly, these transplanted cells seem to exhibit metastatic behavior: these cells are capable of migrating away from the site of initial tumor growth, can migrate through a number of cell layers, and can form secondary colonies [71,73]. Transplantation of tissue possessing mutations for asymmetric division is also a contributing factor for genome instability: alterations in centrosome morphology and number are observed in addition to cytologically abnormal karyotypes [72,73]. These observations clearly demonstrate a commonality - the disruption of asymmetric division in neuroblasts, resulting in the overgrowth of self-renewing daughter cells at the expense of neuronal daughters.

The missegregation of apically- and/or basally localized proteins is a major causal factor for neoplastic growth and tumorigenesis in neuroblasts. Take, for instance, brain tissue possessing neuroblasts with mutated versions of Pins, Miranda, Numb, or Prospero, all crucial asymmetric cell division proteins: transplantation experiments with such brain tissue invariably results in inappropriate neuroblast overproliferation and gives rise to a cancer-like phenotype and ultimately, death [74]. Interestingly, one particular study generated a Drosophila transplantation model of neural stem cell-derived cancer [75]. RNAi-mediated knockdown of cell fate determinants Numb, Brat, and Prospero in neuroblasts resulted in neoplastic tumor formation after transplantation [75]. Additionally, a plethora of studies have demonstrated that absence or disruption of proper cell-fate determinant function (Numb, Pros, Brat) results in an uncontrollable expansion of the neuroblast/progenitor pool, and heavy (or complete) depletion of neuronally committed cells [25,26,28-31,34].

Mutations in three genes in particular, Dlg, Lgl, and Scribble (Scrib), also results in the inability of cell fate determinants to localize asymmetrically in neuroblasts $[30,52,53,57]$. These mutations were also responsible for inducing the formation of neoplastic tumors within the nervous system $[30,52,53,57]$. As was previously mentioned, Lgl in particular serves to restrict aPKC to the self-renewing apical daughter cell [57]. Simultaneous disruption of both Pins and Lgl proteins results in unrestricted growth of neuroblasts due to the delocalization of aPKC [57]. Results from this same study demonstrated that overexpression of a membrane-targeted form of aPKC results in a significant increase in number of neuroblasts [57]. The opposite is also true: loss or reduction of aPKC expression results in a corresponding reduction of neuroblast numbers $[57,76]$.

Because aPKC clearly serves an important role in maintaining a balance between neuroblast self-renewal and differentiation, it is logical to determine which molecules or factors regulate aPKC itself. Two previously mentioned proteins, Protein phosphatase PP2A and the zinc finger protein known as Zif, have been shown to negatively regulate aPKC $[27,55]$. Zif binds to a region of $\mathrm{aPKC}$, thereby repressing aPKC transcription [55]. There is, however, a more complicated feedback mechanism between $\mathrm{aPKC}$ and Zif that has yet to be completely elucidated in the context of regulation of neuroblast self-renewal. As these studies have shown, it is therefore of utmost importance that these determinants (and their adaptor proteins) segregate properly so as to maintain 
the pool of GMCs that eventually give rise to the differentiating neurons of the developing nervous system.

Just as the loss of segregating determinants leads to overgrowth and tumor formation, so too will the loss of machinery controlling mitotic spindle orientation. Several studies have demonstrated that improper mitotic spindle orientation causes improper segregation of proteins that are normally asymmetrically localized. The improper segregation of said proteins in turn leads to unrestricted neuroblast division and outgrowth. As was previously mentioned, loss of Mud, a protein crucial for orienting the mitotic spindle, results in abnormal proliferation of neuroblasts [40-42]. Additionally, neuroblasts possessing double mutations for $L g l$ and Pins, as well as $D l g / G \beta \gamma$ double mutants (GBy is a cortically localized protein that also regulates spindle orientation), demonstrate significant overproliferation relative to wild-type $[57,77]$.

Additionally, in a 2009 study, Cabernard and Doe attempted spindle disruption without altering cell polarity via live imaging of polarity markers and spindle orientation over a time period of multiple divisions, then analyzed cell fate utilizing molecular markers [78]. Results from this study suggest that when the spindle is oriented orthogonally to apical-basal polarity, the cell-fate determinants fail to localize symmetrically rather than asymmetrically, and both daughter cells ultimately form neuroblasts [78]. Maintaining mitotic spindle orientation is thus crucial for maintaining the neuroblast population, ensuring that differentiating neurons are formed and preventing unwanted tumor formation.

Most recently, a protein known as Huntingtin (abbreviated $\mathrm{Htt}$ ) has been shown to regulate mitotic spindle orientation in Drosophila neuroblasts as well as in mammalian cortical progenitor cells [79]. Htt is mutated in Huntington's disease, a neurodegenerative disorder caused by a genetic defect on chromosome 4 . Godin et al showed that RNAi-mediated knockdown of Htt prevents proper spindle orientation; this occurs due to the incorrect localization of the $\mathrm{p} 150^{\text {Glued }}$ subunit of dynactin, dynein, and the NuMA protein [79]. In addition to demonstrating its role in controlling mitosis, further elucidation of Htt's role may hold great promise in the therapeutic treatment of Huntington's disease.

Evidence is emerging that cell cycle regulators play an important role in the division of Drosophila neuroblasts, and disrupting their function may give rise to tumorigenesis. Cell cycle genes have been shown to act as tumor suppressors, whose loss of function manifests in the inability to carry out asymmetric protein localization [80]. Mutations in cell cycle-related genes has also been shown to affect the specification of daughter cell fate, as well as the inappropriate self-renewal of neural progenitors rather than differentiation [80]. A crucial study that first began to characterize the involvement of cell cycle regulators in asymmetric cell division focused on the role of a dominant-negative allele of $\mathrm{Cdc} 2$, known as $c d c 2, c d c 2^{E 51 Q} 76$. In this study, a genetic screen was utilized to identify mutations that inappropriately transformed asymmetric GMC divisions into symmetric divisions, which produced two identical neuronal daughter cells at the expense of GMC daughters. Cdc's normal function is to form a complex with cyclins in order to activate CDK1, a kinase required to force cells from G2 phase to mitosis. In the case of the $c d c 2, c d c 2^{E 51 Q}$ mutant isolated from the genetic screen, both apical and basal proteins involved in neuroblast asymmetric division were unable to localize symmetrically, resulting in the conversion of asymmetric division to symmetric division [81]. Furthermore, utilization of a temperature-sensitive $C d c$ mutant, in which proper Cdc function was weakened, generated the same results [81]. This study clearly demonstrated that cell cycle genes and the proteins they encode should be taken into account when analyzing the underlying mechanisms of excessive cellular growth.

Two other cell cycle regulators are Aurora-A (abbreviated Aur-A) and Polo, which are serine/threonine kinases. Aur-A has been shown to inhibit the selfrenewal of Drosophila larval neuroblasts and promote neuronal differentiation [25]. Mutational analyses demonstrate that Aur-A mutant neuroblasts undergo unrestricted self-renewal, and this phenotype is due to both abnormal aPKC/Numb cortical polarity and misalignment of the mitotic spindle [25]. Another study corroborates these findings: an excessive number of neuroblasts is observed in Aur-A mutants, thereby showing that Aur-A acts as a tumor suppressor [26]. A later study revealed the molecular mechanism for the asymmetric localization of Numb: Aur-A phosphorylates Par-6, which in turn activates aPKC and phosphorylates Lgl; this even allows Bazooka to enter the complex, thereby allowing aPKC to regulate Numb localization to one side of the cell cortex [60]. Polo is another cell cycle regulator that has a hand in asymmetric cell division. Like Aur-A, Polo also acts as a tumor suppressor: excessive neuroblast numbers are observed in Polo mutants [28]. Wang and colleagues have demonstrated that Polo phosphorylates Partner of Numb (Pon), and this is important for Pon to be able to localize Numb [28].

What must also be taken into account are the phosphatases that counteract the effects of the aforementioned kinases. Protein phosphatase 4A (PP4A) and protein phosphatase 2A (PP2A) also play a role in the division of Drosophila neuroblasts. The mechanisms by which these phosphatases operate to regulate are more complicated [61-63] and will not be discussed here; for more details refer to the review by Chang et al [64]. 
As was mentioned earlier, the missegregation of apically- and/or basally-localized proteins is tantamount in ensuring that the asymmetric division machinery function without fail. Slack and colleagues have elegantly demonstrated that anaphase-promoting complex/cyclosome $(\mathrm{APC} / \mathrm{C})$ function is necessary for the proper asymmetric localization of Miranda in particular, as well as its cargo proteins [82]. The $\mathrm{APC} / \mathrm{C}$ is a protein complex whose function is required in the context of the cell cycle; it has been shown to possess roles in destruction of mitotic cyclins, regulation of DNA replication, centrosome, duplication, and mitotic spindle assembly [83-86]. Other recent studies have demonstrated that $\mathrm{APC} / \mathrm{C}$ also has a hand in non-cell cycle-related functions [87-89]. Most relevant to this discussion, Slack et al demonstrated that when any of the core components of $\mathrm{APC} / \mathrm{C}$ are mutated, Miranda and its cargo proteins, Prospero, Brat and Staufen, are unable to properly localize, and instead localize in a pericentrosomal region [82]. Miranda possesses a putative $\mathrm{APC} / \mathrm{C}$ motif at its $\mathrm{C}$-terminal region; disruption or absence of this region results in an inability to ubiquitinate Miranda [82]. Further analysis showed that $\mathrm{APC} / \mathrm{C}$ may facilitate ubiquitination of Miranda, and that this ubiquitination event may be required for the proper asymmetric localization of this protein in dividing neuroblasts [82]. Although much progress has been made in terms of understanding how cell cycle is coupled to asymmetric division machinery, a more widespread and thorough analysis should be performed to identify candidate cell cycle genes involved asymmetric cell division.

\section{Concluding remarks}

Although asymmetric cell division plays a role in the developmental processes of many organisms, it has been best studied in the context of neurogenesis in Drosophila neuroblasts. While the molecules and proteins that play roles in setting up cortical polarity and spindle orientation have been heavily studied and documented, the utilization of neuroblasts as a model to study uncontrolled stem cell self-renewal, and ultimately, tumorigenesis, is relatively new. It is clear that impinging upon any of these components of the asymmetric division machinery will have dire consequences. Transplantation models such as that utilized by Laurenson et al are likely to provide insights into the connection between asymmetric division and tumorigenesis.

A "big picture" approach to understanding the carefully controlled balance between neural stem cell self-renewal and differentiation has utilized transgenic RNAi on the genome-wide level to identify over 600 genes that may control this self-renewal vs. differentiation switch [90]. Knockdown of key genes, such as transcription factors Lola, Ssrp and Barc, results in defective neuroblast lineages. The identification of genes in this study should be further studied with the objective of creating functional gene networks that interact or influence asymmetric cell division machinery.

Despite the advances that have been made in this field of research, there are many questions that have yet to be answered. For one, the exact time point at which neuroblasts re-enter the cell cycle at the end of the embryonic stage remains unclear. Additionally, one must consider the fact that asymmetric cell division in mammalian systems opens a whole new door of complexity: although there may be a high degree of conservation between Drosophila and mammalian homologs, the roles that these mammalian homologs play may be not at all similar to that of the cell fate determinants and proteins in Drosophila. Gaining an understanding of the similarities and differences between Drosophila neuroblasts and mammalian neural progenitors may hold the key to understanding and/or treating various neurological degenerative disorders.

\section{Competing interests}

The authors declare that they have no competing interests.

\section{Authors' contributions}

Both CSKK and WL drafted the manuscript. Both authors read and approved the final manuscript.

Received: 2 September 2012 Accepted: 5 November 2012

Published: 14 November 2012

\section{References}

1. Horvitz HR, Herskowitz I: Mechanisms of asymmetric cell division: two Bs or not two Bs, that is the question. Cell 1992, 68:237-255.

2. Li L, Xie T: Stem cell niche: structure and function. Annu Rev Cell Dev Biol 2005, 21:605-631.

3. Betschinger J, Knoblich JA: Dare to be different: asymmetric cell division in Drosophila, C. elegans and vertebrates. Curr Biol 2004, 14:R674-R685.

4. $\mathrm{Yu} F, \mathrm{KuO} C \mathrm{~T}$, Jan YN: Drosophila neuroblast asymmetric cell division: recent advances and implications for stem cell biology. Neuron 2006, 51:13-20.

5. Gotz M, Huttner WB: The cell biology of neurogenesis. Nat Rev Mol Cell Biol 2005, 6:777-788

6. Kriegstein A, Alvarez-Buylla A: The glial nature of embryonic and adult neural stem cells. Annu Rev Neurosci 2009, 32:149-184.

7. Fortini ME: Notch signaling: the core pathway and its posttranslational regulation. Dev Cell 2009, 16:633-647.

8. Kageyama R, Ohtsuka T, Shimojo H, Imayoshi I: Dynamic regulation of Notch signaling in neural progenitor cells. Curr Opin Cell Biol 2009, 21:733-740.

9. Kopan R, llagan MX: The canonical Notch signaling pathway: unfolding the activation mechanism. Cell 2009, 137:216-233.

10. Bertrand N, Castro DS, Guillemot F: Proneural genes and the specification of neural cell types. Nat Rev Neurosci 2002, 3:517-530.

11. Ross SE, Greenberg ME, Stiles CD: Basic helix-loop-helix factors in cortical development. Neuron 2003, 39:13-25.

12. Lai EC, Deblandre GA, Kintner C, Rubin GM: Drosophila neuralized is a ubiquitin ligase that promotes the internalization and degradation of delta. Dev Cell 2001, 1:783-794.

13. Lai EC, Rubin GM: Neuralized is essential for a subset of Notch pathway-dependent cell fate decisions during Drosophila eye development. Proc Natl Acad Sci USA 2001, 98:5637-5642.

14. Lai EC, Rubin GM: neuralized functions cell-autonomously to regulate a subset of notch-dependent processes during adult Drosophila development. Dev Biol 2001, 231:217-233.

15. Pavlopoulos E, Pitsouli C, Klueg KM, Muskavitch MA, Moschonas NK, Delidakis C: neuralized Encodes a peripheral membrane protein involved in delta signaling and endocytosis. Dev Cell 2001, 1:807-816. 
16. Yeh E, Dermer M, Commisso C, Zhou L, McGlade CJ, Boulianne GL: Neuralized functions as an E3 ubiquitin ligase during Drosophila development. Curr Biol 2001, 11:1675-1679.

17. Honjo T: The shortest path from the surface to the nucleus: RBPkappa/Su(H) transcription factor. Genes Cells 1996, 1:1-9.

18. Kageyama R, Ohtsuka T, Kobayashi T: The Hes gene family: repressors and oscillators that orchestrate embryogenesis. Development 2007 134:1243-1251.

19. Monastirioti M, Giagtzoglou N, Koumbanakis KA, et al: Drosophila Hey is a target of Notch in asymmetric divisions during embryonic and larval neurogenesis. Development 2010, 137:191-201.

20. San-Juan BP, Baonza A: The bHLH factor deadpan is a direct target of Notch signaling and regulates neuroblast self-renewal in Drosophila. Dev Biol 2011, 352:70-82

21. Tio M, Toh J, Fang W, Blanco J, Udolph G: Asymmetric cell division and Notch signaling specify dopaminergic neurons in Drosophila. PLoS One 2011, 6:e26879.

22. Wang W, Liu W, Wang Y, Zhou L, Tang X, Luo H: Notch signaling regulates neuroepithelial stem cell maintenance and neuroblast formation in Drosophila optic lobe development. Dev Biol 2011, 350:414-428.

23. Rhyu MS, Jan LY, Jan YN: Asymmetric distribution of numb protein during division of the sensory organ precursor cell confers distinct fates to daughter cells. Cell 1994, 76:477-491.

24. Le Borgne R, Bardin A, Schweisguth F: The roles of receptor and ligand endocytosis in regulating Notch signaling. Development 2005, 132:1751-1762.

25. Lee CY, Andersen RO, Cabernard C, et al: Drosophila Aurora-A kinase inhibits neuroblast self-renewal by regulating aPKC/Numb cortical polarity and spindle orientation. Genes Dev 2006, 20:3464-3474.

26. Wang H, Somers GW, Bashirullah A, Heberlein U, Yu F, Chia W: Aurora-A acts as a tumor suppressor and regulates self-renewal of Drosophila neuroblasts. Genes Dev 2006, 20:3453-3463.

27. Wang C, Chang KC, Somers G, et al: Protein phosphatase $2 A$ regulates self-renewal of Drosophila neural stem cells. Development 2009, 136:2287-2296.

28. Wang H, Ouyang Y, Somers WG, Chia W, Lu B: Polo inhibits progenitor self-renewal and regulates Numb asymmetry by phosphorylating Pon. Nature 2007, 449:96-100

29. Bello $B$, Reichert $H$, Hirth $F$ : The brain tumor gene negatively regulates neural progenitor cell proliferation in the larval central brain of Drosophila. Development 2006, 133:2639-2648.

30. Betschinger J, Mechtler K, Knoblich JA: Asymmetric segregation of the tumor suppressor brat regulates self-renewal in Drosophila neural stem cells. Cell 2006, 124:1241-1253.

31. Lee CY, Wilkinson BD, Siegrist SE, Wharton RP, Doe CQ: Brat is a Miranda cargo protein that promotes neuronal differentiation and inhibits neuroblast self-renewal. Dev Cell 2006, 10:441-449.

32. Knoblich JA: Mechanisms of asymmetric stem cell division. Cell 2008 132:583-597.

33. Atwood SX, Prehoda KE: aPKC phosphorylates Miranda to polarize fate determinants during neuroblast asymmetric cell division. Curr Biol 2009, 19:723-729.

34. Choksi SP, Southall TD, Bossing T, et al: Prospero acts as a binary switch between self-renewal and differentiation in Drosophila neural stem cells. Dev Cell 2006, 11:775-789.

35. Li L, Vaessin H: Pan-neural Prospero terminates cell proliferation during Drosophila neurogenesis. Genes Dev 2000, 14:147-151.

36. Berger C, Kannan R, Myneni S, Renner S, Shashidhara LS, Technau GM: Cell cycle independent role of Cyclin E during neural cell fate specification in Drosophila is mediated by its regulation of Prospero function. Dev Biol 2010, 337:415-424.

37. Colonques J, Ceron J, Reichert H, Tejedor FJ: A transient expression of Prospero promotes cell cycle exit of Drosophila postembryonic neurons through the regulation of Dacapo. PLoS One 2011, 6:e19342.

38. Zhu Z, Bhat KM: The Drosophila Hem/Kette/Nap1 protein regulates asymmetric division of neural precursor cells by regulating localization of Inscuteable and Numb. Mech Dev 2011, 128:483-495.

39. Nipper RW, Siller KH, Smith NR, Doe CQ, Prehoda KE: Galphai generates multiple Pins activation states to link cortical polarity and spindle orientation in Drosophila neuroblasts. Proc Natl Acad Sci USA 2007, 104:14306-14311.
40. Bowman SK, Neumuller RA, Novatchkova M, Du Q, Knoblich JA: The Drosophila NuMA Homolog Mud regulates spindle orientation in asymmetric cell division. Dev Cell 2006, 10:731-742.

41. Izumi Y, Ohta N, Hisata K, Raabe T, Matsuzaki F: Drosophila Pins-binding protein Mud regulates spindle-polarity coupling and centrosome organization. Nat Cell Biol 2006, 8:586-593.

42. Siller KH, Cabernard C, Doe CQ: The NuMA-related Mud protein binds Pins and regulates spindle orientation in Drosophila neuroblasts. Nat Cell Biol 2006, 8:594-600

43. Siller $\mathrm{KH}$, Doe CQ: Lis1/dynactin regulates metaphase spindle orientation in Drosophila neuroblasts. Dev Biol 2008, 319:1-9.

44. Johnston CA, Hirono K, Prehoda KE, Doe CQ: Identification of an Aurora-A/PinsLINKER/Dlg spindle orientation pathway using induced cell polarity in S2 cells. Cell 2009, 138:1150-1163.

45. Siegrist SE, Doe CQ: Microtubule-induced Pins/Galphai cortical polarity in Drosophila neuroblasts. Cell 2005, 123:1323-1335.

46. Mauser JF, Prehoda KE: Inscuteable regulates the Pins-Mud spindle orientation pathway. PLoS One 2012, 7:e29611.

47. Goldstein B, Macara IG: The PAR proteins: fundamental players in animal cell polarization. Dev Cell 2007, 13:609-622.

48. Suzuki A, Ohno S: The PAR-aPKC system: lessons in polarity. J Cell SCi 2006, 119:979-987

49. Betschinger J, Mechtler K, Knoblich JA: The Par complex directs asymmetric cell division by phosphorylating the cytoskeletal protein Lgl. Nature 2003, 422:326-330.

50. Plant PJ, Fawcett JP, Lin DC, et al: A polarity complex of mPar-6 and atypical PKC binds, phosphorylates and regulates mammalian Lgl. Nat Cell Biol 2003, 5:301-308.

51. Yamanaka T, Horikoshi Y, Sugiyama Y, et al: Mammalian Lgl forms a protein complex with PAR- 6 and aPKC independently of PAR-3 to regulate epithelial cell polarity. Curr Biol 2003, 13:734-743.

52. Ohshiro T, Yagami T, Zhang C, Matsuzaki F: Role of cortical tumour-suppressor proteins in asymmetric division of Drosophila neuroblast. Nature 2000, 408:593-596.

53. Peng CY, Manning L, Albertson R, Doe CQ: The tumour-suppressor genes Igl and dlg regulate basal protein targeting in Drosophila neuroblasts. Nature 2000, 408:596-600.

54. Smith CA, Lau KM, Rahmani Z, et al: aPKC-mediated phosphorylation regulates asymmetric membrane localization of the cell fate determinant Numb. EMBO J 2007, 26:468-480.

55. Chang KC, Garcia-Alvarez G, Somers G, et al: Interplay between the transcription factor Zif and aPKC regulates neuroblast polarity and self-renewal. Dev Cell 2010, 19:778-785.

56. Hafer N, XU S, Bhat KM, Schedl P: The Drosophila CPEB protein Orb2 has a novel expression pattern and is important for asymmetric cell division and nervous system function. Genetics 2011, 189:907-921.

57. Lee CY, Robinson KJ, Doe CQ: Lgl, Pins and aPKC regulate neuroblast self-renewal versus differentiation. Nature 2006, 439:594-598.

58. Speicher S, Fischer A, Knoblich J, Carmena A: The PDZ protein Canoe regulates the asymmetric division of Drosophila neuroblasts and muscle progenitors. Curr Biol 2008, 18:831-837.

59. Carmena A, Makarova A, Speicher S: The Rap1-Rgl-Ral signaling network regulates neuroblast cortical polarity and spindle orientation. J Cell Biol 2011, 195:553-562

60. Wirtz-Peitz F, Nishimura T, Knoblich JA: Linking cell cycle to asymmetric division: Aurora-A phosphorylates the Par complex to regulate Numb localization. Cell 2008, 135:161-173.

61. Chabu C, Doe CQ: Twins/PP2A regulates aPKC to control neuroblast cell polarity and self-renewal. Dev Biol 2009, 330:399-405.

62. Krahn MP, Egger-Adam D, Wodarz A: PP2A antagonizes phosphorylation of Bazooka by PAR-1 to control apical-basal polarity in dividing embryonic neuroblasts. Dev Cell 2009, 16:901-908.

63. Ogawa $H$, Ohta N, Moon W, Matsuzaki F: Protein phosphatase $2 A$ negatively regulates aPKC signaling by modulating phosphorylation of Par-6 in Drosophila neuroblast asymmetric divisions. J Cell Sci 2009, 122:3242-3249.

64. Chang KC, Wang C, Wang H: Balancing self-renewal and differentiation by asymmetric division: insights from brain tumor suppressors in Drosophila neural stem cells. BioEssays 2012, 34:301-310.

65. Schweisguth F: Regulation of notch signaling activity. Curr Biol 2004, 14:R129-R138 
66. Berdnik D, Torok T, Gonzalez-Gaitan M, Knoblich JA: The endocytic protein alpha-Adaptin is required for numb-mediated asymmetric cell division in Drosophila. Dev Cell 2002, 3:221-231.

67. Bhat KM, Gaziova I, Katipalla S: Neuralized mediates asymmetric division of neural precursors by two distinct and sequential events: promoting asymmetric localization of Numb and enhancing activation of Notch-signaling. Dev Biol 2011, 351:186-198.

68. Morin X, Bellaiche Y: Mitotic spindle orientation in asymmetric and symmetric cell divisions during animal development. Dev Cell 2011, 21:102-119.

69. Siller $\mathrm{KH}$, Doe $\mathrm{CQ}$ : Spindle orientation during asymmetric cell division. Nat Cell Biol 2009, 11:365-374.

70. Kraut R, Chia W, Jan LY, Jan YN, Knoblich JA: Role of inscuteable in orienting asymmetric cell divisions in Drosophila. Nature 1996, 383:50-55.

71. Gonzalez C: Spindle orientation, asymmetric division and tumour suppression in Drosophila stem cells. Nat Rev Genet 2007, 8:462-472.

72. Beaucher $M$, Hersperger E, Page-McCaw A, Shearn A: Metastatic ability of Drosophila tumors depends on MMP activity. Dev Biol 2007, 303:625-634.

73. Caussinus E, Gonzalez C: Induction of tumor growth by altered stem-cell asymmetric division in Drosophila melanogaster. Nat Genet 2005 , 37:1125-1129.

74. Zhong W, Chia W: Neurogenesis and asymmetric cell division. Curr Opin Neurobiol 2008, 18:4-11.

75. Laurenson A, Saini N, Jiang Y, Reichert H: Targeted transgenic RNAi knockdown of cell fate determinants induces neoplastic tumor growth and metastasis in a Drosophila transplantation model of neural stem cell derived cancer. J Stem Cell Res Ther 2012, S12:8.

76. Rolls MM, Albertson R, Shih HP, Lee CY, Doe CQ: Drosophila aPKC regulates cell polarity and cell proliferation in neuroblasts and epithelia. J Cell Biol 2003, 163:1089-1098.

77. Kitajima A, Fuse N, Isshiki T, Matsuzaki F: Progenitor properties of symmetrically dividing Drosophila neuroblasts during embryonic and larval development. Dev Biol 2010, 347:9-23.

78. Cabernard C, Doe CQ: Apical/basal spindle orientation is required for neuroblast homeostasis and neuronal differentiation in Drosophila. Dev Cell 2009, 17:134-141.

79. Godin JD, Colombo K, Molina-Calavita M, et al: Huntingtin is required for mitotic spindle orientation and mammalian neurogenesis. Neuron 2010, 67:392-406

80. Chia W, Somers WG, Wang H: Drosophila neuroblast asymmetric divisions: cell cycle regulators, asymmetric protein localization, and tumorigenesis. J Cell Biol 2008, 180:267-272.

81. Tio M, Udolph G, Yang X, Chia W: cdc2 links the Drosophila cell cycle and asymmetric division machineries. Nature 2001, 409:1063-1067.

82. Slack C, Overton PM, Tuxworth RI, Chia W: Asymmetric localisation of Miranda and its cargo proteins during neuroblast division requires the anaphase-promoting complex/cyclosome. Development 2007, 134:3781-3787.

83. Peters JM: The anaphase promoting complex/cyclosome: a machine designed to destroy. Nat Rev Mol Cell Biol 2006, 7:644-656.

84. Sigrist $S$, Jacobs $H$, Stratmann R, Lehner CF: Exit from mitosis is regulated by Drosophila fizzy and the sequential destruction of cyclins $A, B$ and B3. EMBO J 1995, 14:4827-4838.

85. Zur A, Brandeis M: Securin degradation is mediated by fzy and fzr, and is required for complete chromatid separation but not for cytokinesis. EMBO J 2001, 20:792-801.

86. Leismann O, Lehner CF: Drosophila securin destruction involves a D-box and a KEN-box and promotes anaphase in parallel with Cyclin A degradation. J Cell Sci 2003, 116:2453-2460.

87. Konishi Y, Stegmuller J, Matsuda T, Bonni S, Bonni A: Cdh1-APC controls axonal growth and patterning in the mammalian brain. Science 2004, 303:1026-1030.

88. Juo P, Kaplan JM: The anaphase-promoting complex regulates the abundance of GLR-1 glutamate receptors in the ventral nerve cord of C. elegans. Curr Biol 2004, 14:2057-2062.

89. Rappleye CA, Tagawa A, Lyczak R, Bowerman B, Aroian RV: The anaphase-promoting complex and separin are required for embryonic anterior-posterior axis formation. Dev Cell 2002, 2:195-206.

90. Neumuller RA, Richter C, Fischer A, Novatchkova M, Neumuller KG, Knoblich JA: Genome-wide analysis of self-renewal in Drosophila neural stem cells by transgenic RNAi. Cell Stem Cell 2011, 8:580-593.

doi:10.1186/2045-3701-2-38

Cite this article as: Kelsom and Lu: Uncovering the link between malfunctions in Drosophila neuroblast asymmetric cell division and tumorigenesis. Cell \& Bioscience 2012 2:38.

\section{Submit your next manuscript to BioMed Central and take full advantage of:}

- Convenient online submission

- Thorough peer review

- No space constraints or color figure charges

- Immediate publication on acceptance

- Inclusion in PubMed, CAS, Scopus and Google Scholar

- Research which is freely available for redistribution 